\title{
Factores influyentes en el aprendizaje del inglés en instituciones públicas
}

\section{Influencing factors in English learning in public schools}

\author{
Flor Mildred Gonzales Barbarán ${ }^{1}$ \\ Aracelli del Carmen Gonzales Sánchez ${ }^{2}$ \\ Nancy Rita Trujillo Mariño ${ }^{3}$ \\ Carlos Mario Fernández Díaz ${ }^{4}$
}

Recibido: 20 de diciembre de 2019. Aceptado: 14 de enero de 2020

\section{RESUMEN}

El aprendizaje es el punto focal en el proceso de enseñanza y aprendizaje, y por tanto es la preocupación de los docentes cuando los estudiantes no logran internalizar el proceso. Este ensayo aborda algunos de los factores que inciden en el aprendizaje del inglés. Se trabajó con una muestra de 284 estudiantes y se le aplicaron tres encuestas, estas se refieren a la motivación, la percepción del estudiante respecto al desempeño del docente y las estrategias de aprendizaje y para medir el conocimiento del inglés se le aplico un test. Se analizó los datos, obteniendo como resultado que los tres factores en conjunto inciden en el aprendizaje del inglés en un $23.1 \%$, esto nos demuestra que hay que mejorar en muchos aspectos para que los aprendizajes sean efectivos e identificar las otras variables que influyen en los aprendizajes.

Palabras claves: aprendizaje del inglés; motivación; desempeño docente; estrategia de aprendizaje.

\section{ABSTRACT}

Learning is the focal point in the teaching and learning process, and therefore is the concern of teachers when students fail to internalize the process. This essay addresses some of the factors that affect English learning. We worked with a sample of 284 students and three surveys were applied, these refer to motivation, student perception regarding teacher performance and learning strategies and to measure knowledge of English a test was applied. The data was analyzed, obtaining as a result that the three factors together affect the learning of English by $23.1 \%$, this shows us that we must improve in many aspects so that the learning is effective and identify the other variables that influence the learnings.

Keywords: English learning; motivation; teaching performance; learning strategy.

1 Universidad Cesar Vallejo. Correo electrónico: flormildred@gmail.com, ORCID: https://orcid.org/0000-0002-3870-2409

2 Universidad César Vallejo. Correo electrónico: aracelligs@hotmail.com, ORCID: https://orcid.org/0000-0003-0028-9177

3 Universidad César Vallejo. Correo electrónico: ritatruji19@gmail.com, ORCID: https://orcid.org/0000-0003-1951-9366

4 Universidad Peruana de Ciencias Aplicadas. Correo electrónico: pccicfer@upc.edu.pe, ORCID: https://orcid.org/0000-0001-6774-8839

(c) 2020 Revista Multi-Ensayos. 


\section{INTRODUCCIÓN}

El inglés es de suma importancia porque es el idioma que está a la vanguardia de los avances científicos y tecnológicos en el mundo. Su aprendizaje es relevante y los gobiernos de diferentes países de habla hispana u otro idioma han implementado programas para la mejora de los aprendizajes, tal es el caso del Perú que ha implementado "Ingles, puertas al mundo" este programa tiene como propósito que los estudiantes desarrollen competencias comunicativas, esto con el fin de facilitar el acceso a mejores oportunidades académicas y contribuir al desarrollo del país. A pesar de estos programas, estos objetivos no se están cumpliendo por una serie de factores, una de ellas es por la mínima cantidad de horas destinadas al aprendizaje del idioma en las escuelas públicas del Perú, asimismo el entorno social y familiar del estudiante son factores que inciden en los aprendizajes.

El aprendizaje del inglés por lo tanto se convierte en un reto para el estudiante, no solo por ser un idioma extranjero sino por las barreras que se enfrentan para su aprendizaje. Dentro del campo académico hay dos formas de aprender el inglés, la primera de ellos es la adquisición, este proceso se da en forma natural e inconsciente porque el sujeto está inmerso en el ambiente, mientras que el aprendizaje es un proceso consciente y voluntario. Sin embargo, los pobres resultados académicos de los estudiantes en el inglés, plantea interrogantes como ¿Cuáles son los factores que influyen en el aprendizaje del inglés? Para dar respuesta a esta pregunta, fue necesario revisar la literatura científica, las investigaciones realizadas y el dialogo con los estudiantes sobre sus opiniones acerca de las dificultades que enfrentan, después del análisis respectivo se priorizo la motivación, el desempeño docente y las estrategias de aprendizaje como algunos de los factores influyentes en el aprendizaje. Por lo tanto, el objetivo de este estudio es determinar cómo estos factores pueden afectar o favorecer el aprendizaje del idioma, para ello se planteó hipótesis con cada uno de los factores.

\section{DESARROLLO}

El aprendizaje de una lengua es un proceso complejo que permite a la persona dominar un código lingüístico distinto al propio a través de diferentes procesos. Las teorías que fundamentan el aprendizaje de un idioma extranjero devienen desde hace muchas décadas. Uno de ellos es Krasher (1987) que fundamenta que la adquisición de una lengua es un proceso lento donde el aprendiz desarrolla la habilidad de comunicarse luego de que haya desarrollado la habilidad auditiva durante un periodo de tiempo y lo que producen los estudiantes es la consecución del aprendizaje. Es adquirida naturalmente a través de la asimilación de la información comprensible. Se produce la evolución cuando los procesos de aprendizaje se fundamentan en actividades de comunicación espontánea y significativa.

Desde otra perspectiva, el aprendizaje de una lengua es un aprendizaje social porque el estudiante necesita estar en un entorno real o simulado y en contacto con el idioma para comprenderlo, aprenderlo y utilizarlo, esta idea es acorde con lo que señala Vygotsky (1962) donde enfatiza la importancia de la mediación por ser un mecanismo que considera las actividades importantes para la construcción del conocimiento. El docente cumple un rol fundamental en los aprendizajes, por lo tanto, la enseñanza de 
un idioma desde la visión socio cultural no solo es el material lingüístico, sino que el estudiante es el centro de atención, por lo tanto, el docente debe propiciar un ambiente adecuado y debe desarrollar pensamientos en esa nueva lengua. Los estudiantes son capaces de completar tareas sin apoyo a medida que van desarrollando su zona potencial.

\section{Enfoque teórico de la Motivación}

El ser humano por naturaleza tiene necesidades básicas que satisfacer, conforme va desarrollando su pensamiento sus necesidades va creciendo y su estimulación para conseguirlo, estas son denominadas motivación, uno de los teóricos es Maslow (1967) que a través de una figura de pirámide señaló la importancia de las necesidades humanas, que deben ser satisfechas en orden secuencial desde el cimiento hasta la cumbre. Gbollie y Keamu (2017) suscriben que la motivación es un impulso propio, emoción o deseo que mueve a uno hacia particular acción y un objetivo propio, es una receta fundamental para el éxito académico porque involucra factores internos y externos que estimulan el deseo y la energía en las personas para que estén constantemente interesados y comprometidos con alcanzar sus metas.

De igual forma Gardner (1985) indica que la motivación consiste en tres componentes diferentes: esfuerzo, deseo y actitudes favorables. Cuando se amalgaman, estos tres elementos contribuyen en gran medida a la verdadera motivación y destaca la importancia del deseo de alcanzar la meta, así como las actitudes favorables hacia su logro. Por ello los estudiantes tienen que construir y desarrollar las competencias necesarias que le permitan lograr la consecución de sus metas y la voluntad es el grado de motivación de los estudiantes y su deseo de aprender.

\section{Enfoque teórico del desempeño docente}

El papel del docente ha cambiado en las últimas décadas, desde el transmisor de conocimientos, hasta facilitador, Vygotsky lo convierte en un mediador a una figura esencial en el aprendizaje, no solo es transmisor de conocimientos sino que sube de escala, el docente debe ser capaz de propiciar un ambiente adecuado a sus estudiantes y propiciar pensamiento propio en sus estudiantes, a su vez debe conocer los estilos y ritmos de aprendizaje de cada uno de sus estudiantes para que el aprendizaje sea efectivo. Por otro lado, Dover, Rosales y Pérez (2019) señala que el docente de lengua extranjera maximiza las oportunidades de aprendizaje, proporciona la interacción, promueve la consciencia lingüística, contextualiza la entrada lingüística, avala la importancia social y crea consciencia cultural, por lo tanto, las posibilidades se amplían para construir y materializar procesos educativos con significado, pertinencia y congruencia.

Por otro lado, Oxford y Crookall (1989) menciona que el docente debe enfocarse en los estudiantes, y proporcionarles una adecuada estrategia, las técnicas de enseñanza que emplee será un paso fundamental para que consiga la transición de un enfoque centrado en el educador al enfoque centrado en el educando en el aprendizaje de una segunda lengua y logren desarrollar la metacognición del estudiante, lo que les facilitará la selección de las estrategias más adecuadas para la tarea dada. 


\section{Fundamentos teóricos de las Estrategias de Aprendizaje}

Desde el enfoque educativo las estrategias son la ruta que sigue el docente para enseñar y lo que sigue el estudiante para aprender, eso va ir de acuerdo a sus intereses, el ambiente que lo rodea y las herramientas con que cuenta, por consiguiente, Medina \& Nagamine (2019) lo definen como toma de decisiones y dominio procedimental del estudiante que deberá seleccionar para hacer la tarea. Dependerán de las características de la situación concreta de aprendizaje, objetivo el logro del aprendizaje y adquisición de competencias. Para Wang \& Lorenzo (2019). Las estrategias de aprendizaje en lenguas extranjeras son acciones concretas que un estudiante realiza para hacer más viable, autorregulado y transferible a situaciones nuevas, el aprendizaje de un idioma.

Mientras que otros autores como Khokhar \& Sangi (2019) definen las estrategias de aprendizaje como conductas específicas que a menudo toman los educandos conscientemente para mejorar la adquisición, almacenamiento, retención, recuerdo y el uso de nueva información. Para Oxford (2011) y Cohen (1998) las estrategias se deben a variaciones en naturaleza y enfoque y lo clasifica en estrategia de aprendizaje, estrategia de aprendizaje cognitivo, estrategia de desempeño y la estrategia afectiva. Definir las estrategias desde la práctica pedagógica es complejo, las estrategias son las acciones en que cada uno de los estudiantes realiza teniendo en cuenta sus características personales y este va a utilizar las herramientas que tiene a la mano de acuerdo a su realidad, y debe ser capaz de encontrar su propio camino para que pueda interiorizar los aprendizajes por ello la importancia del uso adecuado de las herramientas para lograr sus aprendizajes.

\section{RESULTADOS}

Para sustentar la teoría se decide indagar la influencia de los factores mencionados en estudiantes de cuarto y quinto año del nivel secundario de escuelas públicas, se les aplicó tres encuestas uno por cada factor como la motivación, percepción del estudiante respecto al desempeño docente, estrategias de aprendizaje y un test para medir su conocimiento del inglés. Estas encuestas fueron aplicadas en un solo momento a una muestra de 284 estudiantes.

El test denominado The MET Go! Sample Test (version for 2018) que se les aplicó a los estudiantes para medir su conocimiento del inglés consta de 30 ítems y se evalúa el vocabulario, la estructura gramatical y la comprensión de textos.

\begin{tabular}{|c|c|c|}
\hline & Test de & \\
\hline & $f$ & $\%$ \\
\hline Logrado & 84 & 29.6 \\
\hline No logrado & 200 & 70.4 \\
\hline Total & 284 & 100.0 \\
\hline
\end{tabular}

Tabla 1. Instrumento 1 . MET GO 
Observando el cuadro respectivo de los hallazgos del instrumento, causa preocupación que solo el 29.6\% de los estudiantes lograron responder correctamente el examen mientras que un $70.4 \%$ de los estudiantes no obtuvieron un resultado satisfactorio, estos resultados indican que los estudiantes no están asimilando adecuadamente el idioma. El inglés es un reto para la mayoría de los estudiantes que no están en contacto permanente con el idioma a pesar que tienen acceso al internet. Para otros estudiantes el inglés es solo un curso que tienen que aprobar, pero no le prestan mayor interés. El estudiante tiene que entender que es responsable de su propio aprendizaje y no cambien de actitud es poco probable que logren desarrollar las capacidades comunicativas e lingüísticas por más esfuerzo y dedicación que el docente brinde.

\begin{tabular}{lcrr}
\hline & \multicolumn{3}{c}{ Motivación } \\
\cline { 2 - 4 } & $\mathbf{f}$ & \% & \\
\hline Adecuado & 143 & 50.4 \\
Poco adecuado & 127 & 44.7 \\
Inadecuado & 14 & 4.9 \\
Total & 284 & 100.0 \\
\hline
\end{tabular}

Tabla 2. Instrumento 2 Motivación

En tanto en el segundo instrumento, este evalúa el nivel de motivación con respecto al aprendizaje del inglés, los resultados nos indican que un poco más de la mitad de los estudiantes tienen un nivel adecuado de motivación, pero por otro lado hay un $44.7 \%$ de los estudiantes que su motivación es poco adecuada, y un 4.9\% de los estudiantes su motivación es inadecuado. La razón de estos resultados parte de la realidad del estudiante, del papel que desempeña el docente y de la actitud del estudiante. Pero la motivación va más allá que el simple interés, es poner empeño y voluntad para satisfacer un bien superior, esto implica tener mayores oportunidades en el campo académico, laboral y social.

\begin{tabular}{lcc}
\hline & \multicolumn{3}{c}{ Desempeño docente } \\
\cline { 2 - 3 } & $\mathbf{f}$ & $\%$ \\
\hline Adecuado & 160 & 56.3 \\
Poco adecuado & 109 & 38.4 \\
Inadecuado & 15 & 5,3 \\
\hline Total & 284 & 100.0 \\
\hline
\end{tabular}

Tabla 3. Instrumento 3 desempeño docente

El tercer instrumento trata de medir la percepción del estudiante del desempeño docente de su profesor de inglés, De esta manera, se puede observar que solo un $56.3 \%$ de los estudiantes considera que el trabajo didáctico de su profesor de inglés es adecuado, igualmente, hay un $38.4 \%$ de los estudiantes que consideran que el desempeño de su profesor es poco adecuado y un $5.3 \%$ del estudiante que considera que el papel que desempeña el profesor es inadecuado. Estos resultados nos llevan a conjeturar que el papel que cumple el maestro puede ser causal del pobre desempeño del estudiante en el aprendizaje del inglés. 
Por lo tanto el docente debe mejorar su desempeño en el aula para efectivizar los aprendizajes, sin embargo desde nuestra experiencia docente hay muchas causas del porque el profesor no está cumpliendo adecuadamente su rol, esto va desde el bajo salario hasta las leyes y normas legales que emite el estado sean poco favorables para al docente, sin tomar en cuenta que el exceso de responsabilidades, el entorno familiar, social y económico afecte al docente y no esté motivado para brindar lo mejor de sí hacia sus estudiantes.

\begin{tabular}{lcr}
\hline & \multicolumn{3}{c}{ Estrategias de aprendizaje } \\
\cline { 2 - 4 } & $\mathbf{f}$ & \% \\
\hline Adecuado & 46 & 16,2 \\
Poco adecuado & 196 & 69,0 \\
Inadecuado & 42 & 14,8 \\
Total & 284 & 100.0 \\
\hline
\end{tabular}

Tabla 4. Instrumento 4. Estrategias de aprendizaje

El último instrumento que se les aplicó a los estudiantes fue sobre el uso de las estrategias de aprendizaje, estos hallazgos tratan de explicar del porque los estudiantes no hacen suya el aprendizaje del inglés, como observamos solo un $16.2 \%$ de los estudiantes utiliza efectivamente las estrategias de aprendizaje, mientras que un $69.0 \%$ de los estudiantes el uso de las estrategias de aprendizaje es poco adecuado y un $14.8 \%$ de los estudiantes no utilizan adecuadamente las estrategias de aprendizaje.

Las estrategias de aprendizaje es la forma como utiliza las herramientas y materiales que tiene a su mano para aprender un determinado tema o concepto y hacerlo suyo, según los resultados el estudiante están presentando dificultades en la aplicación adecuada de las estrategias, por ello deben cambiar la forma de ejecutarlo o aplicar otras estrategias, los estudiantes cuentan con recursos tecnológicos que les permite acceder a todo tipo de información en el idioma, y el docente debe brindar la información necesaria del uso y considerar en sus sesiones la aplicación de estos recursos para motivarlos en sus aprendizajes.

\begin{tabular}{|c|c|c|c|c|c|}
\hline Hipótesis & Factores & $\mathbf{N}$ & Sig. bilateral & Nagelkerke & Influencia \\
\hline $\begin{array}{l}\text { Hipótesis } \\
\text { General }\end{array}$ & $\begin{array}{l}\text { Motivación-Desempeño } \\
\text { docente-Estrategias de } \\
\text { aprendizaje*Aprendizaje del inglés }\end{array}$ & 284 & 0.000 & 0,231 & Nivel bajo $23,1 \%$ \\
\hline $\begin{array}{l}\text { Hipótesis } \\
\text { Especifica } 1\end{array}$ & Motivación*Aprendizaje del inglés & 284 & 0.000 & 0,102 & Nivel muy bajo $10,2 \%$ \\
\hline $\begin{array}{l}\text { Hipótesis } \\
\text { Especifica } 2\end{array}$ & $\begin{array}{l}\text { Desempeño docente* Aprendizaje del } \\
\text { inglés }\end{array}$ & 284 & 0,000 & 0,126 & Nivel muy bajo $12,6 \%$ \\
\hline $\begin{array}{l}\text { Hipótesis } \\
\text { Especifica } 3\end{array}$ & $\begin{array}{l}\text { Estrategia de aprendizaje * } \\
\text { Aprendizaje del inglés }\end{array}$ & 284 & 0,000 & 0.129 & Nivel muy bajo $12,9 \%$ \\
\hline
\end{tabular}

Tabla 5. Nivel de influencia de los factores

Para medir el nivel de influencia de los factores en conjunto y en forma individual se aplicó el modelo de regresión logística, este permite evaluar la influencia de cada una de las variables independientes 
sobre la variable respuesta y controlar el efecto del resto, en la tabla 5 las variables independientes son la motivación del estudiante, el desempeño docente y las estrategias de aprendizaje y la variable respuesta es el aprendizaje del inglés que se evaluó mediante un test. Como se observa la significancia bilateral es 0.000 donde indica que el modelo es adecuado y que las variables en conjunto influyen en el aprendizaje del inglés. El índice de Nagelkerke es 0,231 esto nos indica que 23,1\% del aprendizaje del inglés es explicada por las variables incluidas en el modelo, pero hay un 76,9\% que son explicadas por otras variables no identificadas.

En cuanto a la hipótesis especifica uno, referente a la motivación con el aprendizaje del inglés, la significancia bilateral es 0,000 donde indica que la motivación influye en el aprendizaje del inglés. Pero solo en un $10,2 \%$. La hipótesis especifica dos, referente al desempeño docente con el aprendizaje del inglés la significancia bilateral es 0,000 que indica que el desempeño docente influye en el aprendizaje del inglés en un $12.6 \%$. La hipótesis especifica tres, referida a las estrategias de aprendizaje con el aprendizaje del inglés la significancia es 0,000 que indica la estrategia de aprendizaje influye en el aprendizaje del inglés en un nos indica que el 12,9\%.

Ante estos resultados es que el aprendizaje es el eje principal en el ámbito educativo, a partir de ella han surgido diversas teorías desde la conductista hasta la cognitivista tratando de explicar cómo el sujeto asimila y se apropia de ese nuevo conocimiento. El aprendizaje implica la adquisición de conocimiento y como se construye. Desde el enfoque de Vygotsky el estudiante es el principal protagonista en su aprendizaje, pero este no aprende solo, sino que requiere interactuar con el profesor y sus compañeros para que el conocimiento logre una significancia, y que la zona de desarrollo potencial sea estimulada activamente por el estudiante.

El rol del docente va más allá de la mediación, deja de ser un portador de conocimientos para asumir un rol diferente y mejorar la calidad de su intervención en el proceso de aprendizaje. El docente no solo suministra un nuevo idioma, sino que le ayuda a desarrollar pensamientos e ideas en esa lengua. El estudiante será capaz de ejecutar tareas y acciones conforme va desarrollando su zona de desarrollo próximo y aproximándose al potencial mientras que las estrategias benefician al estudiante en el uso de técnicas que aportan en la adquisición de conocimientos, habilidades, o destrezas para desarrollar las competencias lingüístico-comunicativas adecuadas. Asimismo, motivación es el impulso que mueve al individuo hacia el logro de sus objetivos sean personales, académicos y/o personales.

\section{CONCLUSIÓN}

Después de una revisión exhaustiva de la teoría, de estudios previos y la participación de los estudiantes para determinar qué factores puedan tener una influencia en el aprendizaje del inglés, se priorizó que la motivación del estudiante, desempeño docente y las estrategias de aprendizaje pueden ser considerados como factores influyentes, para el análisis estadístico respetivo se utilizó el modelo de regresión múltiple, dicho modelo, se encontró que estas variables en conjunto explican un 23,1\% en el aprendizaje del inglés. Asimismo, cada una de estas variables por separado tienen una influencia muy baja en el aprendizaje del 
inglés, como por ejemplo la motivación influye en el aprendizaje del inglés, pero solo en un 10,2\%. De acuerdo a la teoría, el rol del docente es una de los factores fundamentales en el proceso de aprendizaje del estudiante, al hacer el análisis respectivo se determinó que el factor de desempeño docente explica solo el $12.6 \%$ el aprendizaje del inglés y la influencia de las estrategias de aprendizaje con el aprendizaje del inglés es $12,9 \%$.

Desde la perspectiva docente, hay varias estrategias que implementar para mejorar los aprendizajes en nuestros estudiantes, desde una planificación curricular adecuada y acorde a la realidad de sus estudiantes hasta la evaluación del mismo en todo el proceso para poder darle los ajustes necesarios, este trabajo debe ser compartido con otros docentes del área y de la institución.

\section{REFERENCIAS}

Cohen, A. (1998). Strategies in Learning and Using Second Language. The Electronic Journal of English as a Second Language, 3(4).

Dover, Y., Rosales, A., \& Pérez, R. (2019). Construcción de una metodología para el aprendizaje del inglés en contextos rurales: Contribución de aprendientes. Revista Electrónica Educare, 23(1), 1-24. EISSN: 1409-4258 doi: http://dx.doi.org/10.15359/ree.23-1.14

Gardner, 1985 - Gardner, R.C. (1985). Social psychology and second language learning: The role of attitudes and motivation. London: Edward Arnold Publishers

Gbollie, C., \& Keamu, H. (2017).Student academic performance: The role of motivation, strategies, and perceived factors hindering Liberian junior and senior high school students learning. Education Research International, Volume 2017, Article ID 1789084, 11 pages https://doi. org/10.1155/2017/1789084.

Khokhar, S., \& Sangi, M. (2019). Language Learning Strategies And Styles Of Second Language Learners. Grassroots, 52(1). ISSN: 2521-456x (Online), ISSN:1726-0396(Print).

Maslow, A. (1991). Motivación y personalidad. Madrid: Díaz de Santos S.A.

Medina, D., \& Nagamine, M. (2019). Estrategias de aprendizaje autónomo en la comprensión lectora de estudiantes de secundaria. Propósitos y Representaciones, 7(2), 134-159. doi: http://dx.doi. org/10.20511/pyr2019.v7n2.276

Oxford, R. (2011). Teaching and Researching Language Learning Strategies. Harlow: Pearson Longman.

Oxford, R. \& Crookall. (1989). Research on language learning strategies: Methods, findings, and instructional issues. The Modern Language Journal, 73 (4), 404-419.

Wang, L., \& Lorenzo, M. T. C. (2019). Factores y estrategias del aprendiz preadolescente en chino como lengua extranjera. Estudio de caso en España. Onomázein: Revista de lingüística, filología y traducción de la Pontificia Universidad Católica de Chile, (43), 158-175. ISSN-e 0717-1285 ISSN: 0718-5758 